\title{
Biotransformation of Furosemide in Kidney Transplant Patients
}

\author{
D.E. Smith ${ }^{1,2}$ and L.Z. Benet ${ }^{1}$ \\ 'Department of Pharmacy, School of Pharmacy, University of California, San Francisco, California and \\ ${ }^{2}$ College of Pharmacy, The University of Michigan, Ann Arbor, Michigan, USA
}

Summary. The metabolic fate of furosemide was studied in kidney transplant patients after oral and intravenous administration of the diuretic at therapeutic doses. Serial urine samples were collected over a $24 \mathrm{~h}$ period and furosemide was analyzed by a specific high performance liquid chromatographic method using fluorescence detection. We found no evidence of the putative furosemide metabolite, 2-amino-4-chloro-5-sulfamoylanthranilic acid (CSA), in any of the samples analyzed. The amount of furosemide excreted as the glucuronide metabolite accounted for $8 \%$ of the available dose, whether administered orally or by intravenous infusion. In addition, the significant positive correlation observed between the percent of the available dose excreted as furosemide glucuronide and the renal clearance of furosemide $(r=0.581, p<0.02)$ suggests that the glucuronidation process for furosemide may be occurring in the kidney. Furosemide and its glucuronide metabolite accounted for only $45 \%$ of the intravenous dose recovered in the urine. Biliary excretion of unchanged furosemide and/or furosemide glucuronide into the feces probably accounts for the remainder of the dose not recovered.

Key words: furosemide, kidney transplant patients; metabolism, renal function, furosemide glucuronide, biliary excretion

The metabolism of furosemide has been studied in healthy volunteers and in various patient populations [1, 2], but not in kidney transplant patients. In addition, the data concerning the metabolic fate of furosemide is controversial, as reviewed in References [1] and [3]. While some investigators have re- ported 2-amino-4-chloro-5-sulfamoylanthranilic acid (CSA) to be a metabolite of furosemide, others have not confirmed this finding. A glucuronide metabolite of furosemide has also been reported, although poorly quantitated in some studies, and found to vary with dosing history and renal function in others. These discrepancies may reflect problems inherent in the assay procedures as reported by Smith et al. [3]. The present investigation was, therefore, undertaken in order to define, in kidney transplant patients, the metabolism of furosemide using a specific high performance liquid chromatographic (HPLC) assay.

\section{Materials and Methods}

Furosemide tablets ( $40 \mathrm{mg}$, lot \# 601549) and intravenous solution $(10 \mathrm{mg} / \mathrm{ml}$, lot \#'s X61922 and 613379) were obtained from Hoechst-Roussel Pharmaceuticals, Inc. (Somerville, NJ). All other drugs and reagents used in the analytical methods were identical to those previously described $[3,4]$.

\section{Patient Studies}

Characteristics of the 8 kidney transplant patients studied were previously described and the pharmacokinetics and pharmacodynamics of furosemide in this patient population were evaluated [4]. After an overnight fast, each patient received either an oral or intravenous dose of furosemide at approximately 8 a.m. Furosemide tablets were taken with water or fruit juice while the solution was infused intravenously over a $10 \mathrm{~min}$ period. In addition, patients fasted for at least $2 \mathrm{~h}$ after oral administration of furosemide. Voided urine was collected hourly from $1 \mathrm{~h}$ prior to dosing until $8 \mathrm{~h}$ after dosing, and then 
Table 1. Urinary excretion ${ }^{\mathrm{a}}$ of furosemide and its conjugated metabolite in kidney transplant patients

\begin{tabular}{|c|c|c|c|c|}
\hline Patient & Treatment & $\begin{array}{l}\text { Unchanged } \\
\text { furosemide } \\
\text { [mg] }\end{array}$ & $\begin{array}{l}\text { Conjugated } \\
\text { furosemide } \\
{[\mathrm{mg}]}\end{array}$ & FGL [\%] \\
\hline \multirow[t]{2}{*}{$\mathrm{CT}$} & 160 mg p.o. & 21.2 & 3.2 & 2.7 \\
\hline & $160 \mathrm{mg}$ i. v. & 28.2 & 7.6 & 4.8 \\
\hline \multirow[t]{2}{*}{ EH } & $120 \mathrm{mg}$ p.o. & 9.7 & 3.6 & 9.9 \\
\hline & $120 \mathrm{mg} \mathrm{i} . \mathrm{v}$. & 32.6 & 9.7 & 8.1 \\
\hline \multirow[t]{2}{*}{$\mathrm{DH}$} & 120 mg p.o. & 31.0 & 8.9 & 9.1 \\
\hline & $120 \mathrm{mg}$ i.v. & 41.8 & 8.4 & 7.0 \\
\hline \multirow[t]{2}{*}{ LT } & $120 \mathrm{mg}$ p.o. & 17.2 & 1.8 & 3.5 \\
\hline & $120 \mathrm{mg}$ i. v. & 43.6 & 5.9 & 4.9 \\
\hline \multirow[t]{2}{*}{ SJ } & $120 \mathrm{mg}$ p.o. & 16.1 & 2.4 & 5.2 \\
\hline & $120 \mathrm{mg}$ i. $\mathrm{v}$. & 40.3 & 2.4 & 2.0 \\
\hline \multirow[t]{2}{*}{ PD } & $40 \mathrm{mg}$ p.o. & 8.5 & 2.9 & 15.0 \\
\hline & $40 \mathrm{mg}$ i.v. & 16.1 & 3.9 & 9.8 \\
\hline \multirow[t]{2}{*}{ WJ } & $80 \mathrm{mg}$ p.o. & 31.9 & 4.5 & 10.3 \\
\hline & $80 \mathrm{mg}$ i.v. & 56.7 & 8.2 & 10.2 \\
\hline \multirow[t]{4}{*}{ FR } & $80 \mathrm{mg}$ p.o. & 20.7 & 6.0 & 13.6 \\
\hline & $80 \mathrm{mg}$ i.v. & 34.6 & 8.5 & 10.6 \\
\hline & & $\begin{array}{c}\text { Mean } \\
\pm \text { SD }\end{array}$ & p.o. & $\begin{array}{c}8.7 \\
(4.5)\end{array}$ \\
\hline & & $\begin{array}{c}\text { Mean } \\
\pm S D\end{array}$ & i.v. & $\begin{array}{c}7.2 \\
(3.1)\end{array}$ \\
\hline
\end{tabular}

${ }^{a}$ Collection period of $24 \mathrm{~h} ;{ }^{\text {b }}$ Values were previously reported [4];

c Expressed in furosemide weight units

pooled from 8 to $24 \mathrm{~h}$. The kidney transplant patients were studied on consecutive days after oral and intravenous dosing of furosemide, respectively. All patients signed the 'Consent Form' approved by the Human Research Committee of the University of California, San Francisco.

\section{Analytical Methods}

Urine samples were measured in parallel for unchanged furosemide and its glucuronide metabolite using HPLC with fluorescence detection as previously described [3]. Duplicate urine samples $(0.20 \mathrm{ml})$ were prepared and added to $0.80 \mathrm{ml}$ buffer solutions $(0.1 \mathrm{M}$ sodium acetate $\mathrm{pH} 4.5)$, one containing 2000 units $\beta$-glucuronidase and the other containing only buffer. The difference in furosemide concentration between urine samples with and without $\beta$-glucuronidase was used as a measure of the metabolite concentration (expressed in furosemide weight units). Complete conversion of furosemide glucuronide to the parent drug was demonstrated since identical results were obtained over a 100 -fold enzyme concentration range (100-10,000 units/ $0.20 \mathrm{ml}$ urine sample).

Urine samples were analyzed for CSA by HPLC with fluorescence detection as previously reported [3]. The sensitivity for this direct injection method is $2.5 \mu \mathrm{g} / \mathrm{ml}$ using $0.05 \mathrm{ml}$ urine samples.

\section{Calculations}

The percent of the available dose of furosemide excreted as the glucuronide metabolite, FGL, was estimated by:

$$
\begin{aligned}
& \mathrm{FGL}=100 \times\left[\left(\mathrm{Ae}^{\infty}\right) \text { enzyme }-\left(\mathrm{Ae}^{\infty}\right) \text { no enzyme }\right] \\
& \mathrm{F} \times \text { DOSE }
\end{aligned}
$$

where the amount of unchanged drug recovered in the urine at time infinity after treatment with $\beta$-glucuronidase is represented by $\left(\mathrm{Ae}^{\infty}\right)$ enzyme. The amount of unchanged drug recovered in the urine after treatment with buffer is represented by $\left(\mathrm{Ae}^{\infty}\right)$ no enzyme. The extent of availability, F, was previously reported [4] for oral doses in these patients and assumed to be equal to one for all intravenous doses. The above equation assumes a negligible first-pass effect for the metabolism of furosemide to its glucuronide metabolite, as will be discussed subsequently.

\section{Results}

The putative metabolite of furosemide, CSA, was sought in the urine samples for all 8 kidney transplant patients after both oral and intravenous administration. We found no evidence of this metabolite in any of the samples analyzed.

Table 1 lists the urinary excretion of furosemide and its glucuronide metabolite after both oral and intravenous treatments. Urinary recovery of the parent compound and metabolite accounted for only $45.2 \pm 17.4 \%$ of the intravenous dose. The amount of furosemide excreted as the glucuronide metabolite was approximately $8 \%$ of the available dose and varied considerably between patients, as reflected by a coefficient of variation of almost $50 \%$. Nevertheless, in Fig. 1 a significant positive correlation was observed between the $\%$ of the available dose excreted as furosemide glucuronide and the renal clearance of furosemide in the 8 kidney transplant patients studied $(r=0.581 ; p<0.02)$. In addition, no significant difference was found in the $\%$ of the available dose excreted as furosemide glucuronide with respect to route of administration $(8.7 \pm 4.5 \%$ for p.o. versus $7.2 \pm 3.1 \%$ for i.v.; $p>0.10$; paired $t$-test). In our previous report [4] the patients listed in Table 1 were defined as furosemide responders (patients SJ, PD, WJ and FR) and non-responders based on the dose of drug required to elicit the desired diuretic effect. Although there was a tendency for an increased \% of the available dose to be excreted as furosemide glucuronide in the responders $(9.6 \pm 4.2 \%$ versus $6.3 \pm 2.7 \%)$, this difference was not significant $(p>$ 0.05 ; unpaired $t$-test). 


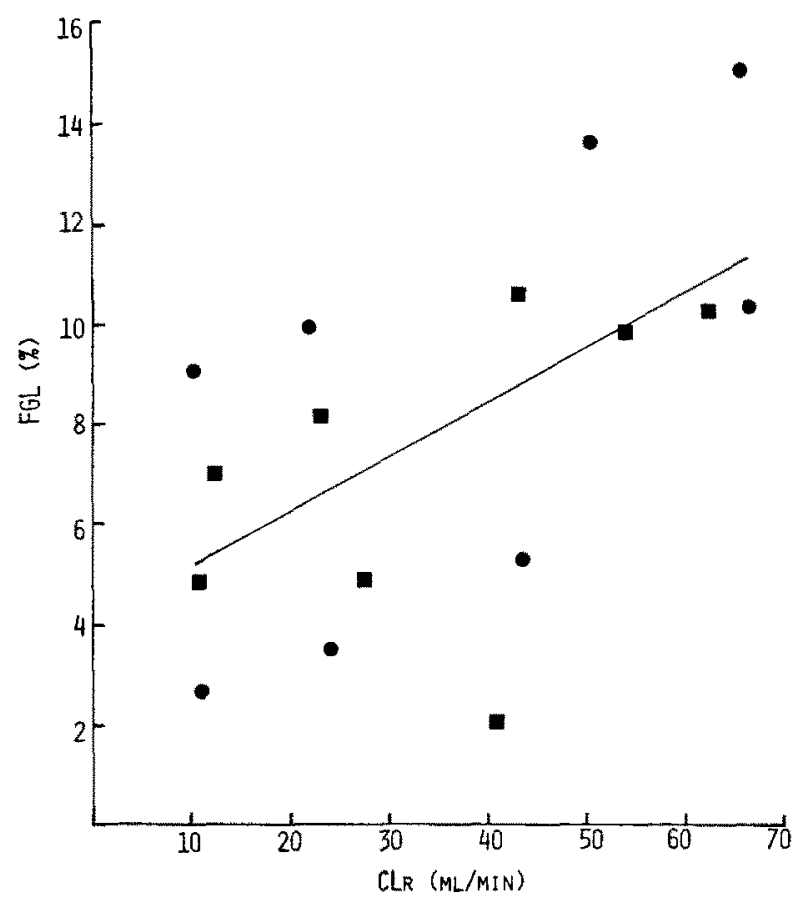

Fig. 1. Correlation between the percent of the available dose of furosemide excreted as glucuronide metabolite (FGL) and furosemide renal clearance (CLr) after oral (@) and intravenous (E) dosing of furosemide to kidney transplant patients $(r=0.581$, $p<0.02$ )

\section{Discussion}

Conflicting results concerning the metabolism of furosemide to CSA probably reflect problems inherent in the assay method. Smith et al. [3] clearly demonstrate that CSA is not formed in vivo, but is an analytical artifact formed during an acid extraction procedure. Using a direct injection assay method, without prior extraction and/or derivatization, Smith et al. [3] found no evidence of CSA in 9 healthy volunteers studied following intravenous and oral dosing. In the present study, the putative metabolite of furosemide, CSA, was not detected in the urine samples of kidney transplant patients. This finding supports the contention [3] that CSA is not a metabolite of furosemide.

The significant positive correlation between the $\%$ of the available dose excreted as furosemide glucuronide and the renal clearance of furosemide (Fig.1) suggests that the biotransformation of furosemide to its glucuronide metabolite may be occuring in the kidney. This hypothesis is supported by the fact that the ratio of the amount of furosemide glucuronide to unchanged furosemide in the urine is similar for kidney transplant patients $(0.23 \pm 0.10$ for p. $0 . ; 0.21 \pm 0.08$ for i.v.) and healthy volunteers
$(0.22 \pm 0.05$ for p.o.; $0.21 \pm 0.04$ for i.v.; [3]). In addition, no significant difference was observed in the $\%$ of the available dose of furosemide excreted as glucuronide metabolite, whether the drug was administered orally or intravenously to kidney transplant patients and healthy volunteers. This implies that the first-pass effect for hepatic and gut wall metabolism to its glucuronide metabolite is probably negligible. This is consistent with the recent finding of Verbeeck et al. [5] which indicates that the liver does not play a significant role in the nonrenal clearance of furosemide in the dog. In contrast to the results reported here, Andreasen et al. [6] demonstrated a highly significant negative correlation between drug serum clearance and the fraction of furosemide excreted as glucuronide in patients with severe arterial hypertension, which would seem to indicate that greater metabolism results from prolonged drug residence in the body. Our calculations of their data show that a highly negative correlation also exists between renal clearance and the fraction excreted as furosemide glucuronide. However, these earlier results may be influenced by analytical problems since more recently Andreasen et al. [7] reported furosemide glucuronide levels in healthy volunteers which were in marked contrast to the data published earlier using TLC separation.

Total urinary recovery of furosemide and its glucuronide metabolite accounted for only $45 \%$ of the intravenous dose (versus $80 \%$ in normal volunteers). The remainder of the dose was probably excreted into the feces via the biliary route, either as unchanged furosemide and/or furosemide glucuronide. This is consistent with previous studies $[8,9]$ which demonstrate that over $60 \%$ of furosemide- $S^{35}$ can be recovered in the feces after intravenous administration of drug to patients with impaired renal functions.

Acknowlegdements. We are indebted to Dr. J.G. Gambertoglio and Dr. F. Vincenti for their initiative and provision of the patient population for these studies. The authors are most appreciative of the help given by Ms. J.Almeida in preparation of the manuscript.

This work was supported in part by NIH Grant AM 20884 and by the Earl C. Anthony Fund. During the course of this work D.E.S. was supported as an NIH Predoctoral Scholar on NIH Training Grant GM 07175.

\section{References}

1. Benet LZ (1979) Pharmacokinetics/pharmacodynamics of furosemide in man: A review. J Pharmacokinet Biopharm 7:1-27 
2. Cutler RE, Blair AD (1979) Clinical pharmacokinetics of furosemide. Clin Pharmacokinet 4: 279-296

3. Smith DE, Lin ET, Benet LZ (1980) Absorption and disposition of furosemide in healthy volunteers, measured with a metabolite specific assay. Drug Metab Dispos 8: 337-342

4. Smith DE, Gambertoglio JG, Vincenti F, Benet LZ (1981) Furosemide kinetics and dynamics after kidney transplant. Clin Pharmacol Ther 30: 105-113

5. Verbeeck RK, Gerkens JF, Wilkinson GR, Branch RA (1981) Disposition of furosemide in functionally hepatectomized dogs. J Pharmacol Exp Ther 216: 479-483

6. Andreasen F, Pedersen OL, Mikkelsen E (1978) Distribution, elimination and natriuretic effect of furosemide in patients with severe arterial hypertension. Eur J Clin Pharmacol 4: 237-244

7. Andreasen F, Christensen CK, Jakobsen FK, Morgensen CE (1981) The use of HPLC to elucidate the metabolism and urinary excretion of furosemide and its metabolic products. Acta Pharmacol Toxicol 49: 223-229
8. Beermann B, Dalén E, Lindström B (1977) Elimination of furosemide in healthy subjects and in those with renal failure. Clin Pharmacol Ther 22: 70-78

9. Rupp W (1974) Pharmacokinetics and pharmacodynamics of lasix. Scot Med J 19: 5-13

Received: March 9, 1981

in revised form: September 1, 1982

accepted: March 4, 1983

Leslie Z. Benet, Ph.D.

Department of Pharmacy

School of Pharmacy, 926-S

University of California

San Francisco, California 94143, USA 